\title{
Axiomatization of the counting rule for cost-sharing with possibly redundant items
}

\author{
Gildas Sédry Fopa ${ }^{1} \cdot$ Issofa Moyouwou ${ }^{2} \cdot$ Joseph Siani $^{3,4,5}$ D
}

Received: 15 April 2020 / Accepted: 20 September 2021 / Published online: 20 October 2021

(c) The Author(s), under exclusive licence to Springer-Verlag GmbH Germany, part of Springer Nature 2021

\begin{abstract}
For cost allocation problems with an existing set of indivisible public resources with heterogeneous individual needs and non-rivalry access, an axiomatization is provided for the allocation rule that proportionally charges agents for a given resource with respect to their counting liability indices. The main result we obtain holds in the class of cost allocation rules that are additive in cost and simply combines a new independence property together with the well-known axioms of consistency and independence of supplementary items.
\end{abstract}

\section{Introduction}

A cost allocation problem refers to any situation in which some agents have to share the cost of some facilities they jointly benefit from. We consider here the class of allocation problems introduced by Hougaard and Moulin (2014) on how to share the cost of an existing set of indivisible public resources with non-rivalry access and heterogeneous individual needs. For other classes of cost allocation problems,

Joseph Siani

sianijosephr@yahoo.fr

Gildas Sédry Fopa

fopagildas@yahoo.fr

Issofa Moyouwou

imoyouwou2@yahoo.fr

1 Department of Mathematics, Research and Training Unit for Doctorate in Mathematics Computer Sciences and Applications, University of Yaounde I, Yaoundé, Cameroon

2 Department of Mathematics, Advanced Teachers' Training College, University of Yaounde I, Yaoundé, Cameroon

3 IESEG School of Management, UMR 9221 - LEM - Lille Economie Management, 59000 Lille, France

4 Univ. Lille, UMR 9221 - LEM - Lille Economie Management, 59000 Lille, France

5 CNRS, UMR 9221 - LEM - Lille Economie Management, 59000 Lille, France 
the reader is referred to Bergantiños and Martínez (2014) on the problem of allocating the total cost of transport on a given tree among several users; or Thomson (2007) on cost allocation problems that arise in airport games. More examples are presented by Hougaard (2018), Thomson (2001) and Sharkey (1995).

Individual needs or liabilities to resources may differ from one agent to another. Thus designing an allocation rule that takes into account the possibility of heterogeneous individual needs becomes an important issue. In general, there are two normative approaches pursued by theorists to find what rules are reasonable for a given family of problems.

In some contributions, authors focus on a class of solutions described by a given list of compelling properties met only by the rules within that class. This is the case in the paper by Hougaard and Moulin (2014) who axiomatize a family of one-parameter cost ratios for sharing the cost of several indivisible public resources when agents access resources without rivalry; see also Hougaard (2018) for cost allocation problems when resources have limited reliability (each resource may randomly fail or not); and Moulin (2000) or Young (1988) for some families of methods for rationing problems.

In some other contributions, the attention is paid to a given set of desirable requirements that pins down a unique rule. This is for example the case in the papers by Béal et al. (2018); Bergantiños et al. (2014) and Moulin and Laigret (2011). Another famous illustration of the one-problem-one-solution approach is the Shapley value (Shapley , 1953) on cooperative games with transferable utilities. In this paper, we provide a similar investigation for the counting rule introduced by Hougaard and Moulin (2014). The counting rule proportionally charges an agent on a given resource with respect to his/her counting liability, that is the probability that he/she is served by a minimal set of resources that contains the resource under consideration.

To characterize the counting rule among cost allocation rules that are additive in cost, we simply focus on the counting cost ratio associated with the counting rule and introduce an independence axiom: the axiom of Independence of Regular Concatenation $(I R C)$ which is the counterpart, within the current framework, of independence axioms formulated around mergeability for rationing problems, bankruptcy problems or allocation problems in networks; see de Frutos (1999), Moulin (2002, 2013) or Hougaard (2009). It appears that (IRC) together with Consistency, Independence of Supplementary Items, Replication, Neutrality, Anonymity and the four dispositions provided by Hougaard and Moulin (2014, Definition 2) uniquely identify the counting cost ratio. We show that (IRC) also allows a characterization of the counting cost ratio with fewer axioms in our main result.

The remainder of this paper is organized as follows. Section 2 is devoted to notations and definitions. Some key concepts on cost allocation problems under consideration are presented in Sect. 2.1. The counting rule and its cost ratio index are defined in Sect. 2.2; and axioms for cost allocation problems we use are stated and commented in Sect. 2.3: all those axioms are from Hougaard and Moulin (2014); except the Independence of Regular Concatenation and Identity. Results are stated and proved in Sect. 3. We first give in Sect. 3.1 some preliminary results that help 
us in easing the proof of the main result we present in Sect. 3.2. Finally, Sect. 4 concludes the paper.

\section{Cost allocation problems with possibly redundant items}

\subsection{The model}

We consider the model of cost allocation problems presented by Hougaard and Moulin (2014): $\mathcal{R}$ and $\mathcal{N}$ are, respectively, an infinite set of potential resources and an infinite set of potential agents. Agents share access to resources without rivalry. A cost allocation problem is a quadruplet $\left(N, R, \mathcal{D}^{N}, c\right)$ that consists in:

(i) A non empty and finite set $N$ of agents with $N \subset \mathcal{N}$.

(ii) A non empty and finite set $R$ of resources with $R \subset \mathcal{R}$.

(iii) A profile $\mathcal{D}^{N}$ that specifies for each agent $i \in N$ a non empty set $\mathcal{D}^{i}$ of non empty subsets of $R$ such that agent $i$ 's needs are met only when at least one serving set from $\mathcal{D}^{i}$ is provided. The set $\mathcal{D}^{i}$ is the service constraint of agent $i$ and each element of $\mathcal{D}^{i}$ is called a serving set for $i$.

(iv) A vector of cost $c=\left(c_{r}\right)_{r \in R}$ that gives the cost $c_{r} \geq 0$ of each resource $r \in R$. The total cost $c(R)=\sum_{r \in R} c_{r}$ should be shared among agents in $N$.

Individual service constraints are assumed to be inclusion monotonic: for each agent $i, B \in \mathcal{D}^{i}$ whenever $B$ is a superset of a serving set $A \in \mathcal{D}^{i}$. The service needs $\mathcal{D}^{i}$ of agent $i$ is then completely described by the list $\overline{\mathcal{D}}^{i}$ of all $i$ 's minimal serving sets: $A \in \overline{\mathcal{D}}^{i}$ if and only if agent $i$ is served by $A$ but no longer by any proper subset of $A$. A resource $r$ is relevant for agent $i$ if $r \in A$ for some $i$ 's minimal serving set $A$; and irrelevant for agent $i$ otherwise. The set of all resources that are relevant for agent $i$ is denoted by $\mathcal{H}^{i}$; that is $\mathcal{H}^{i}=\cup_{A \in \overline{\mathcal{D}}^{i}} A$. All resources in profile $\mathcal{D}^{N}$ that are not in $\cup_{i \in N} \mathcal{H}^{i}$ are irrelevant for all agents in $N$. Each such resource is called redundant. The set of all agent $i$ 's minimal serving sets that contain resource $r$ is denoted by $\overline{\mathcal{D}}^{i}(r)$; that is $A \in \overline{\mathcal{D}}^{l}(r)$ if, and only, if $A \in \overline{\mathcal{D}}^{i}$ and $r \in A$. Also note that $r$ is relevant for agent $i$ if, and only if, $\overline{\mathcal{D}}^{i}(r) \neq \emptyset$.

In this paper, we say that the service constraint $\mathcal{D}^{i}$ of agent $i$ is of type $p$ if $\left|\overline{\mathcal{D}}^{i}\right|=p$. Moreover, we write $\mathcal{D}^{i} \sim^{r}(q, p)$ if $\left|\overline{\mathcal{D}}_{i}(r)\right|=q$ and $\left|\overline{\mathcal{D}}^{i}\right|=p$. When there is no ambiguity to assuming that the information on $N$ and $R$ is embedded in the description of $\mathcal{D}^{N}$, the cost allocation problem $\left(N, R, \mathcal{D}^{N} \Im\left(\mathcal{D}^{i}\right)_{i \in N}, c\right)$ will simply be represented by $\left(\mathcal{D}^{N}, c\right)$ and the corresponding set of resources by $\mathcal{R}\left(\mathcal{D}^{N}\right)$. Given a resource $r$, the set of all agents in profile $\left(\mathcal{D}^{N}, c\right)$ for whom $r$ is relevant is denoted by $N(r)$. The set of all allocation problems on $\mathcal{N}$ and $\mathcal{R}$ is denoted by $\mathcal{P}(\mathcal{N}, \mathcal{R})$.

Example 2.1 Consider an airport with three runways $r_{1}, r_{2}$ and $r_{3}$ of distinct lengths deserved by three airlines 1,2 and 3 . The service needs of the three airlines are as follows: 1 is served by any of the two runways $r_{2}$ or $r_{3} ; 2$ is served by any two consecutive runways; and 3 is served by the combination of all the three only. 
The profile $\mathcal{D}^{N}$ of airlines' needs is completely described by $\overline{\mathcal{D}}^{1}=\left\{\left\{r_{2}\right\},\left\{r_{3}\right\}\right\}$, $\overline{\mathcal{D}}^{2}=\left\{\left\{r_{1}, r_{2}\right\},\left\{r_{2}, r_{3}\right\}\right\}$ and $\overline{\mathcal{D}}^{3}=\left\{\left\{r_{1}, r_{2}, r_{3}\right\}\right\}$. The corresponding set of resources is $\mathcal{R}\left(\mathcal{D}^{N}\right)=\left\{r_{1}, r_{2}, r_{3}\right\}$.

A cost allocation rule $F$ is a mapping that assigns to each cost allocation problem $\left(\mathcal{D}^{N}, c\right)$ in $\mathcal{P}(\mathcal{N}, \mathcal{R})$, a vector of payments $F\left(\mathcal{D}^{N}, c\right)=\left(F_{i}\left(\mathcal{D}^{N}, c\right)\right)_{i \in N}$ such that

$$
\sum_{i \in N} F_{i}\left(\mathcal{D}^{N}, c\right)=c\left(\mathcal{R}\left(\mathcal{D}^{N}\right)\right)
$$

where

$$
c\left(\mathcal{R}\left(\mathcal{D}^{N}\right)\right)=\sum_{r \in \mathcal{R}\left(\mathcal{D}^{N}\right)} c_{r}
$$

Hereafter, (1) is called the budget-equation of $\operatorname{problem}\left(\mathcal{D}^{N}, c\right)$.

Example 2.2 Define the egalitarian $\operatorname{rule} E$ on $\mathcal{P}(\mathcal{N}, \mathcal{R})$ for all allocation problems $\left(\mathcal{D}^{N}, c\right)$ and for all $i \in N$ by

$$
E_{i}\left(\mathcal{D}^{N}, c\right)=\sum_{r \in \mathcal{H}^{i}} \frac{c_{r}}{|N(r)|}+\sum_{r \in \mathcal{R}\left(\mathcal{D}^{N}\right) \backslash\left(\cup_{j \in N} \mathcal{H}^{j}\right)} \frac{c_{r}}{|N|} .
$$

This cost allocation rule equally splits the cost of each resource $r$ between all agents for whom $r$ is relevant if $r$ is not redundant; and between all agents otherwise. For illustration, if $c_{t}$ is the cost expenditure for runways of type $r_{t}$ in Example 2.1,

$$
\begin{aligned}
& E_{1}\left(\mathcal{D}^{N}, c\right)=\frac{1}{3} c_{2}+\frac{1}{3} c_{3}, E_{2}\left(\mathcal{D}^{N}, c\right)=\frac{1}{2} c_{1}+\frac{1}{3} c_{2}+\frac{1}{3} c_{3} \\
& \text { and } E_{3}\left(\mathcal{D}^{N}, c\right)=\frac{1}{2} c_{1}+\frac{1}{3} c_{2}+\frac{1}{3} c_{3} .
\end{aligned}
$$

\subsection{The counting rule and the counting cost ratio index}

By using the axioms of Additivity, Anonymity, Neutrality, Consistency, Replication, and Irrelevance of Supplementary Items, Hougaard and Moulin (2014) have characterized the one-parameter family $\left(\psi^{\pi}\right)_{\pi>0}$ of cost allocation rules defined for all cost allocation problems $\left(\mathcal{D}^{N}, c\right)$ by

$$
\psi_{i}^{\pi}\left(\mathcal{D}^{N}, c\right)=\sum_{r \in \mathcal{R}\left(\mathcal{D}^{N}\right)} \frac{\theta_{i}^{\pi}\left(\mathcal{D}^{N}, r\right)}{\sum_{j \in N} \theta_{j}^{\pi}\left(\mathcal{D}^{N}, r\right)} c_{r}
$$

where 


$$
\theta_{i}^{\pi}\left(\mathcal{D}^{N}, r\right)=\left(\frac{\left|\overline{\mathcal{D}}^{i}(r)\right|}{\left|\overline{\mathcal{D}}^{i}\right|}\right)^{\pi}
$$

if $r$ is relevant for some agents; and $\theta_{i}^{\pi}\left(\mathcal{D}^{N}, r\right)=1$ otherwise. ${ }^{1}$

The ratio of the cost of resource $r$ supported by agent $i$ in $\mathcal{D}^{N}$ is denoted by $\varphi_{i}^{\pi}\left(\mathcal{D}^{N}, r\right)$ and is, with respect to the cost allocation rule $\psi^{\pi}$, given by

$$
\varphi_{i}^{\pi}\left(\mathcal{D}^{N}, r\right)=\frac{\theta_{i}^{\pi}\left(\mathcal{D}^{N}, r\right)}{\sum_{j \in N} \theta^{\pi}\left(\mathcal{D}^{j}, r\right)} .
$$

The term $\theta^{\pi}\left(\mathcal{D}^{i}, r\right)$ is interpreted as a measure of the liability of agent $i$ to resource $r$ in $\mathcal{D}^{i}$.

In particular, for $\pi=1, \theta^{1}\left(\mathcal{D}^{i}, r\right)$ and $\varphi_{i}^{1}\left(\mathcal{D}^{N}, r\right)$ are respectively named, after Hougaard and Moulin (2014), the counting liability and the counting cost ratio of agent $i$ for $r$. The cost allocation rule $\psi^{1}$ associated with $\varphi^{1}$, is called the counting rule. Moreover, the interpretation of the counting liability is straightforward. In fact, the counting liability $\theta_{i}^{1}\left(\mathcal{D}^{N}, r\right)=\left|\overline{\mathcal{D}}^{i}(r)\right| /\left|\overline{\mathcal{D}}^{i}\right|$ of agent $i$ to a non redundant resource $r$ is simply the ratio of the total number of agent $i$ 's minimal serving sets containing $r$ by the total number of agent $i$ 's minimal serving sets. Moreover, when the information on the final cost vector and the knowledge of each agent on the service needs of other agents are such that no agent can discriminate, in terms of cost, between his/her minimum service sets, each agent, to be served, uniformly selects one of his/ her minimal serving sets. In this case, agent $i$ 's counting liability for $r$ corresponds to the expected rate of use of $r$ by agent $i$; and the sum

$$
\Theta\left(\mathcal{D}^{N}, r\right)=\sum_{i \in N} \theta_{i}^{1}\left(\mathcal{D}^{N}, r\right),
$$

which accounts for the use of resource $r$ by all the agents in the network, can be seen as the overall service demand on resource $r$ given profile $\mathcal{D}^{N}$.

Example 2.3 In Example 2.1, the counting liability of each agent for each runway zone is as follows:

\footnotetext{
${ }^{1}$ To let $\theta_{i}^{\pi}\left(\mathcal{D}^{N}, r\right)$ be defined everywhere, we have extended its definition to redundant resources by assuming that all agents in a profile are equally liable for redundant resources. This does not alter the counting rule since Hougaard and Moulin (2014) also assume that the cost of each redundant resource is equally split among agents.
} 


$$
\begin{array}{lll}
\theta_{1}^{1}\left(\mathcal{D}^{N}, r_{1}\right)=0 & \theta_{1}^{1}\left(\mathcal{D}^{N}, r_{2}\right)=\frac{1}{2} & \theta_{1}^{1}\left(\mathcal{D}^{N}, r_{3}\right)=\frac{1}{2} \\
\theta_{2}^{1}\left(\mathcal{D}^{N}, r_{1}\right)=\frac{1}{2} & \theta_{2}^{1}\left(\mathcal{D}^{N}, r_{2}\right)=1 & \theta_{2}^{1}\left(\mathcal{D}^{N}, r_{3}\right)=\frac{1}{2} \\
\theta_{3}^{1}\left(\mathcal{D}^{N}, r_{1}\right)=1 & \theta_{3}^{1}\left(\mathcal{D}^{N}, r_{2}\right)=1 & \theta_{3}^{1}\left(\mathcal{D}^{N}, r_{3}\right)=1 \\
\Theta\left(\mathcal{D}^{N}, r_{1}\right)=\frac{3}{2} & \Theta\left(\mathcal{D}^{N}, r_{2}\right)=\frac{5}{2} & \Theta\left(\mathcal{D}^{N}, r_{3}\right)=2
\end{array}
$$

When agents are randomly served by their respective minimal serving sets, the expected rates of use of $r_{1}$ are $0, \frac{1}{2}$ and 1 for agents 1,2 and 3 respectively. The counting rule then charges each agent proportionally to his/her expected rate of use of $r_{1}$ in such a way that agents 1,2 and 3 respectively pay 0, one-third and two-third of the cost $c_{1}$ of $r_{1}$. Applying the same analysis to the costs of $r_{2}$ and $r_{3}$ leads to the following individual shares by the counting rule:

$$
\begin{gathered}
\psi_{1}^{1}\left(\mathcal{D}^{N}, c\right)=\frac{1}{5} c_{2}+\frac{1}{4} c_{3}, \quad \psi_{2}^{1}\left(\mathcal{D}^{N}, c\right)=\frac{1}{3} c_{1}+\frac{2}{5} c_{2}+\frac{1}{4} c_{3} \\
\text { and } \psi_{3}^{1}\left(\mathcal{D}^{N}, c\right)=\frac{2}{3} c_{1}+\frac{2}{5} c_{2}+\frac{1}{2} c_{3} .
\end{gathered}
$$

For a non redundant resource, values of $\pi \in[0,1]$ have, as $\pi$ tends to 0 , a flattening effect that emerges at $\pi=0$ to equal shares for all agents $i$ for whom $r$ is relevant; that is

$$
\theta_{i}^{0}\left(\mathcal{D}^{N}, r\right)=\frac{1}{|N(r)|} \quad \text { if } r \in \mathcal{H}^{i} ; \text { and } \theta_{i}^{0}\left(\mathcal{D}^{N}, r\right)=0 \text { otherwise }
$$

Similarly, values of $\pi \in(1,+\infty)$ have, as $\pi$ tends to $+\infty$, an outward effect to let only agents with the maximum counting liability for $r$ to be equally charged for the cost of $r$ at $\pi=+\infty$; by a little abuse of notation, that is

$$
\theta_{i}^{+\infty}\left(\mathcal{D}^{N}, r\right)=\frac{1}{\left|N^{*}(r)\right|} \quad \text { if } \mathcal{D}^{i}=\arg \max \left\{\theta_{j}^{1}\left(\mathcal{D}^{N}, r\right): j \in N\right\} ; \quad \text { and } \theta_{i 1}^{+\infty}\left(\mathcal{D}^{N}, r\right)=0 \text { otherwise }
$$

where $N^{*}(r)$ is the set of all agents with the largest liability index for $r$.

When there is no clear motivation for distorting the counting liability, the choice of $\pi=1$ is natural. Hereafter, we characterize the counting rule $\Phi=\psi^{1}$ trough the mapping $\varphi^{1}$ associated with $\Phi$ and called the counting cost ratio index by Hougaard and Moulin (2014). For simplicity, $\varphi^{1}$ will simply be denoted by $\phi$. Thus, for all allocation problems $\left(\mathcal{D}^{N}, c\right)$ and for all agents $i \in N$ by

$$
\Phi_{i}\left(\mathcal{D}^{N}, c\right)=\sum_{r \in \mathcal{R}\left(\mathcal{D}^{N}\right)} \phi_{i}\left(\mathcal{D}^{N}, r\right) c_{r} .
$$

In general, a cost ratio index is any function $f$ that maps each profile $\mathcal{D}^{N}$ of service needs and each resource $r \in \mathcal{R}\left(\mathcal{D}^{N}\right)$ to a collection $\left(f_{i}\left(\mathcal{D}^{N}, r\right)\right)_{i \in N}$ of non negative numbers that sum to 1 ; that is 


$$
\sum_{i \in N} f_{i}\left(\mathcal{D}^{N}, r\right)=1 .
$$

Condition (6) will be referred to as the budget-balance condition (on resource $r$ ). A cost allocation rule $F$ is associated to the cost ratio index $f$ if for all allocation problems $\left(\mathcal{D}^{N}, c\right)$ and for all agents $i \in N$,

$$
F_{i}\left(\mathcal{D}^{N}, c\right)=\sum_{r \in \mathcal{R}\left(\mathcal{D}^{N}\right)} f_{i}\left(\mathcal{D}^{N}, r\right) c_{r} .
$$

\subsection{Axioms for cost allocation problems}

The first four axioms we consider are from Hougaard and Moulin (2014).

Additivity ( $A D D)$ : A cost allocation rule $F$ is additive (in costs) if for all cost allocation problems $\left(\mathcal{D}^{N}, c\right)$ and for all cost vectors $c^{\prime}=\left(c_{r}^{\prime}\right)_{r \in \mathcal{R}\left(\mathcal{D}^{N}\right)}$ and $c^{\prime \prime}=\left(c_{r}^{\prime \prime}\right)_{r \in \mathcal{R}\left(\mathcal{D}^{N}\right)}$,

$$
F\left(\mathcal{D}^{N}, c\right)=F\left(\mathcal{D}^{N}, c^{\prime}\right)+F\left(\mathcal{D}^{N}, c^{\prime \prime}\right) \text { whenever } c=c^{\prime}+c^{\prime \prime} .
$$

The cost share of each agent in a profile is the sum of his/her cost shares when the cost vector is additively decomposed into two new cost vectors to obtain two allocation problems without any change in the service needs of the agents.

Interestingly, it can be shown that a cost allocation rule $F$ is additive if, and only if, it is associated to some cost ratio index $f$; that is, $F$ is in the form $(7)^{2}$. An additive cost rule $F$ is therefore completely determined by its cost ratio index $f$. The other axioms that follow are stated for a cost ratio index $f$ (and thus for the cost allocation rules $F$ associated with $f$ ).

Consistency $(C O N)$ : For all profiles $\mathcal{D}^{N}$, for all resources $r$ in $\mathcal{D}^{N}$ and for all agents $i \in N$,

$$
f_{j}\left(\mathcal{D}^{N}, r\right)=\left(1-f_{i}\left(\mathcal{D}^{N}, r\right)\right) f_{j}\left(\mathcal{D}^{N \backslash\{i\}}, r\right) \quad \text { for all } j \in N \backslash\{i\} .
$$

Suppose that an agent, say $i$, pays for his/her contribution $f_{i}\left(\mathcal{D}^{N}, r\right) c_{r}$ for each resource $r$ as prescribed by the cost ratio index $f$. Then the cost vector is updated from $c$ to $c^{\prime}$ with $c_{r}^{\prime}=\left(1-f_{i}\left(\mathcal{D}^{N}, r\right)\right) c_{r}$ for each resource $r$ in $\mathcal{D}^{N}$. The cost allocation problem is now $\left(\mathcal{D}^{N \backslash\{i\}}, c^{\prime}\right)$. Being consistent requires that the cost ratio of each agent $j \in N \backslash\{i\}$ for each resource should not change from $\left(\mathcal{D}^{N}, c\right)$ to $\left(\mathcal{D}^{N \backslash\{i\}}, c^{\prime}\right)$.

Given two profiles $\mathcal{D}^{N}$ and $\mathcal{E}^{N}$ of service constraints, an agent $i \in N$, a resource $b$ in $\mathcal{E}^{N}$ is supplementary to agent $i$ 's needs from $\mathcal{D}^{N}$ to $\mathcal{E}^{N}$ if $b$ is not a resource in $\mathcal{D}^{N}$ and for all $A \in \overline{\mathcal{E}}_{i}^{i}, A \backslash\{b\} \in \overline{\mathcal{D}}^{l}$ or $A \in \overline{\mathcal{D}}^{l}$. It can be checked that, this is equivalent to saying that $\overline{\mathcal{E}}$ is obtained from $\overline{\mathcal{D}}^{i}$ by only embedding $b$ to some minimal serving sets in $\overline{\mathcal{D}}^{i}$. Now, the set of resources in $\mathcal{E}^{N}$ consists in all resources in $\mathcal{D}^{N}$ together with $b$.

\footnotetext{
${ }^{2}$ Interested readers are referred to the supplementary material joined to this paper.
} 


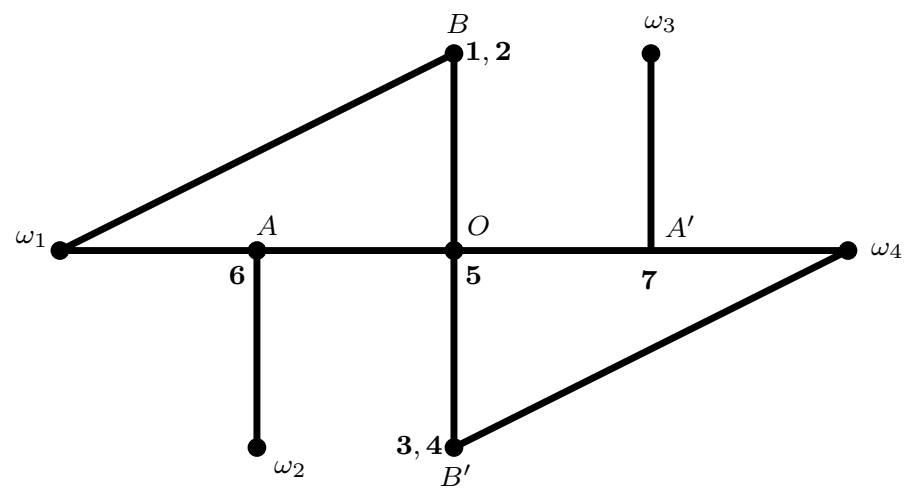

Fig. 1 Facility locations on a graph

Irrelevance of Supplementary Items (ISI): For all profiles $\mathcal{D}^{N}$ and $\mathcal{E}^{N}$, if a resource $b$ in $\mathcal{E}^{N}$ is supplementary to the needs of the agents from $\mathcal{D}^{N}$ to $\mathcal{E}^{N}$, then

$$
f\left(\mathcal{D}^{N}, r\right)=f\left(\mathcal{E}^{N}, r\right) \text { for all resources } r \text { in } \mathcal{D}^{N} .
$$

When from one profile to another, service constraints differ only on a single supplementary resource, then the cost ratio of each agent for all other resources coincides in both profiles.

Relatedness (REL): For all profiles $\mathcal{D}^{N}$, for all non redundant resources $r \in \mathcal{R}\left(\mathcal{D}^{N}\right)$, and for all agents $i \in N$,

$$
f_{i}\left(\mathcal{D}^{N}, r\right)=0 \text { if, and only, if } r \notin \mathcal{H}^{i} .
$$

An agent $i$ contributes nothing for the cost of a non redundant resource $r$ if, and only if, $r$ is irrelevant to $i$. This condition combines two previous requirements from (Hougaard and Moulin 2014, items (ii) and (iii) in Definition 2).

Now, we introduce two new axioms. The first one is a very basic requirement that each cost ratio index should meet.

Identity (IDE): For all profiles $\mathcal{D}^{N}$, if $\mathcal{D}^{i}=\mathcal{D}^{j}$ for all agents $i$ and $j$, then

$$
f_{i}\left(\mathcal{D}^{N}, r\right)=f_{j}\left(\mathcal{D}^{N}, r\right) \text { for all agents } i, j \in N \text { and for all resources } r \in \mathcal{R}\left(\mathcal{D}^{N}\right) .
$$

When all agents have the same needs, they are equally charged for all resources.

Hereafter, any cost ratio index that satisfies $(I D E)$ and $(R E L)$ is called reasonable. To continue, we need a few more notation and definitions. Let $S$ be a finite subset of at least two agents and denote by $\mathcal{D}^{S}=\left(\mathcal{D}^{i}\right)_{i \in S}$ the profile of service constraints of agents in $S$. We say that the needs of agents in $S$ are mergeable if all pairs of agents in $_{i} S$ have non overlapping sets of minimal serving sets; that is for all $\{i, j\} \subseteq S$, $\overline{\mathcal{D}}^{i} \cap \overline{\mathcal{D}}^{J}=\emptyset$. In this case, the concatenation of the service constraints in $\mathcal{D}^{S}$ is the 
service constraint $\mathcal{C}=\mathcal{D}^{[S]}$ such that $\overline{\mathcal{C}}=\cup_{i \in S} \overline{\mathcal{D}}^{i}$. Any concatenation of service constraints of the same type is called regular. Given a profile $\mathcal{D}^{N}$, the profile $\mathcal{D}^{N[S]}$ is obtained from $\mathcal{D}^{N}$ by only replacing the service constraint of each agent $i \in S$ by $\mathcal{C}=\mathcal{D}^{[S]}$ provided that agents in $S$ have mergeable service constraints ${ }^{3}$; more precisely, for $\mathcal{E}^{N}=\mathcal{D}^{N[S]}, \mathcal{E}^{i}=\mathcal{D}^{[S]}$ for all $i \in S$, and $\mathcal{E}^{i}=\mathcal{D}^{i}$ for all $i \in N \backslash S$.

Example 2.4 Consider the graph in Fig. 1 where the costly items are the edges of the graph. Agents live on nodes other than $\omega_{j}, j=1,2,3,4$ which are some facility locations that provide four separate types of services. First assume that agent 1 is served only by $\omega_{1}$ or $\omega_{2}$; while agent 2 is interested only by $\omega_{3}$ or $\omega_{4}$. We simply denote by $X Y$ the edge delimited by $X$ and $Y$. The service needs of 1 and 2 are completely described by

$$
\overline{\mathcal{D}}^{1}=\left\{\left\{B \omega_{1}\right\},\left\{B O, O A, A \omega_{1}\right\},\left\{B O, O A, A \omega_{2}\right\}\right\}
$$

and

$$
\overline{\mathcal{D}}^{2}=\left\{\left\{B O, O A^{\prime}, A^{\prime} \omega_{3}\right\},\left\{B O, O A^{\prime}, A^{\prime} \omega_{4}\right\},\left\{B O, O B^{\prime}, B^{\prime} \omega_{4}\right\}\right\}
$$

respectively. Note that agents 1 and 2 are separately served by two disjoint sets of minimal serving sets.

Suppose that at each facility location, the four types of services are now available. Agents 1 and 2 are now served by any of the four facilities $\omega_{j}$. The service needs of 1 and 2 are thus updated to

$$
\begin{aligned}
\overline{\mathcal{E}}^{1}=\overline{\mathcal{E}}^{2}=\left\{\left\{B \omega_{1}\right\},\left\{B O, O A, A \omega_{1}\right\},\left\{B O, O A, A \omega_{2}\right\},\left\{B O, O A^{\prime}, A^{\prime} \omega_{3}\right\},\left\{B O, O A^{\prime}, A^{\prime} \omega_{4}\right\},\right. \\
\left.\left\{B O, O B^{\prime}, B^{\prime} \omega_{4}\right\}\right\} .
\end{aligned}
$$

Clearly, $\overline{\mathcal{E}}^{1}$ and $\overline{\mathcal{E}}^{2}$ are obtained from $\overline{\mathcal{D}}^{1}$ and $\overline{\mathcal{D}}^{2}$ by a regular concatenation. It can be easily checked that such a change in the service constraints of the two players alters the overall service demand on no resource in the network.

The next axiom requires that a regular concatenation of the service constraints of agents in $S$ should not affect the cost ratio of agents out of $S$.

Independence of regular concatenation (IRC):For all profiles $\mathcal{D}^{N}$, for all non empty subsets $S$ of $N$, if agents in $S$ have mergeable service constraints of the same type, then for all resources $r \in \mathcal{R}\left(\mathcal{D}^{N}\right)$,

$$
f_{k}\left(\mathcal{D}^{N[S]}, r\right)=f_{k}\left(\mathcal{D}^{N}, r\right) \quad \text { for all } k \in N \backslash S .
$$

Suppose that some agents are somehow constrained to use only a subset of the resources, any two of these agents using separate parts of the resources. Then (IRC) requires that whenever all parts have the same size, whether we consider the unrestricted or the restricted case should not matter for the other agents. Intuitively, a

\footnotetext{
${ }^{3}$ In contrast with the usual statement of mergeability, all agents in $S$ remain in the profile.
} 
regular concatenation can be seen as a kind of an update in the service needs of some agents that does not affect the overall service demand on each resource of the network.

Remark 2.1 It can be easily checked that $\phi=\psi^{1}$ is the unique cost allocation index of the form $\psi^{\pi}$ that satisfies (IRC). Therefore the counting ratio index is completely characterized among all cost ratio indices by Consistency, Independence of Supplementary Items, Replication, Neutrality, Anonymity and the four dispositions provided by Hougaard and Moulin (2014) in their Definition 2. We provide below another characterization of the counting ratio index with fewer axioms.

We will sometimes consider situations in which some new resources become complementary to some agents' needs. We say that a non empty finite $X$ of resources is complementary to agent $i$ 's needs from $\mathcal{D}^{i}$ to $\mathcal{E}^{i}$, denoted by $\mathcal{E}^{i}=\mathcal{D}^{i}[X]$, if for all subsets $A$ of resources: $A \in \mathcal{E}^{i}$ if, and only if, $A \backslash X \in \mathcal{D}^{i}$ and $A \cap X \neq \emptyset$. To be satisfied, agent $i$ needs one of his/her serving set in $\mathcal{D}^{i}$ and at least one new resource from $X$. This is illustrated in the following example:

Example 2.5 Before the COVID-19 pandemic, three faculties 1, 2 and 3 had to pay for the costs of three security gate controls $\mathrm{a}, \mathrm{b}$ and $\mathrm{c}$. Gates a and $\mathrm{b}$ gave access to Campus 1 for students from faculty 1 and for some students from faculty 2 . Gate c gave access to Campus 2 for students from faculty 3 and for some students from faculty 2 . The profile $\mathcal{D}^{N}$ of service needs was as follows

$$
\overline{\mathcal{D}}^{1}=\{\{a\},\{b\}\}, \overline{\mathcal{D}}^{2}=\{\{a, c\},\{b, c\}\} \text { and } \overline{\mathcal{D}}^{3}=\{\{c\}\} .
$$

After the first peak of the COVID-19 pandemic, faculties who resumed activities were called to install at least one facility for COVID-19 prevention (stations combining hand-washing points, hydroalcoholic gels, ...). Ten identical such facilities (denoted by $x_{1}, x_{2}, \ldots, x_{10}$ ) were first installed on Campus 1 . The updated profile $\mathcal{E}^{N}$ of service constraints was described by:

$$
\begin{aligned}
& \overline{\mathcal{E}}^{1}=\left\{\left\{a, x_{j}\right\},\left\{b, x_{j}\right\}: 1 \leq j \leq 10\right\}=\mathcal{D}^{1}[X] \\
& \overline{\mathcal{E}}^{2}=\left\{\left\{a, c, x_{j}\right\},\left\{b, c, x_{j}\right\}: 1 \leq j \leq 10\right\}=\mathcal{D}^{2}[X] \\
& \overline{\mathcal{E}}^{3}=\overline{\mathcal{D}}^{1}=\{\{c\}\}
\end{aligned}
$$

where $X=\left\{x_{1}, x_{2}, \ldots, x_{10}\right\}$. It appears that the introduction of $x_{1}, x_{2}, \ldots, x_{10}$ simply resulted in adding one of the ten new resources to a minimal serving sets of agents 1 and 2 without altering the role of each of the previous resources: the set $X$ was clearly complementary to agent 1 's needs from $\mathcal{D}^{1}$ to $\mathcal{E}^{1}$. The same observation occurred for agent 2. Finally, a total of eleven other identical facilities $y_{1}, y_{2}, \ldots, y_{11}$ were installed on Campus 2 and the final profile $\mathcal{T}^{N}$ of service needs described by: 


$$
\begin{aligned}
& \overline{\mathcal{T}}^{1}=\left\{\left\{a, x_{j}\right\},\left\{b, x_{j}\right\}: 1 \leq j \leq 10\right\} \\
& \overline{\mathcal{T}}^{2}=\left\{\left\{a, c, x_{j}, y_{k}\right\},\left\{b, c, x_{j}, y_{k}\right\}: 1 \leq j \leq 10,1 \leq k \leq 11\right\}=\mathcal{E}^{2}[Y] \\
& \overline{\mathcal{T}}^{3}=\left\{\left\{c, y_{k}\right\}: 1 \leq k \leq 11\right\}=\mathcal{E}^{3}[Y]
\end{aligned}
$$

where $Y=\left\{y_{1}, y_{2}, \ldots, y_{11}\right\}$.

As illustrated in the precedent example, when a set $X$ of $p$ resources is complementary to agent $i$ 's needs from $\mathcal{D}^{i}$ to $\mathcal{E}^{i}$, each minimal serving set $A$ of agent $i$ in $\mathcal{D}^{i}$ corresponds to $p$ isomorphic minimal serving sets in $\mathcal{E}^{i}$, each obtained by simply embedding to $A$ one of the $p$ new resources.

Remark 2.2 The reader can easily checked that all cost ratio indices of the form $\varphi^{\pi}$ defined by (3) satisfy $\varphi^{\pi}\left(\mathcal{E}^{N}, r\right)=\varphi^{\pi}\left(\mathcal{D}^{N}, r\right)$ whenever for all agents $i \in N$, $\mathcal{E}^{i}=\mathcal{D}^{i}\left[X_{i}\right]$ for some set $X_{i}$ of resources.

\section{Results}

\subsection{Preliminary results}

We first present some preliminary results on cost ratio indices that satisfy $(C O N)$.

Proposition 3.1 If a reasonable cost ratio index $f$ satisfies $(C O N)$, then for all profiles $\mathcal{D}^{N}$ of service constraints, for all agents $i$ and for all resources $r$ that are relevant to agent $i$,

$$
\frac{f_{j}\left(\mathcal{D}^{N}, r\right)}{f_{i}\left(\mathcal{D}^{N}, r\right)}=\frac{f_{j}\left(\mathcal{D}^{\{i, j\}}, r\right)}{f_{i}\left(\mathcal{D}^{\{i, j\}}, r\right)} \quad \text { for all } \quad j \in N \backslash\{i\} .
$$

Proof Consider a reasonable cost ratio index $f$ that meets $(C O N)$, a profile $\mathcal{D}^{N}$ of service constraints, an agent $i$ and a resource $r$ that is relevant to agent $i$. Let $j \in N \backslash\{i\}$. Note that $f$ is reasonable by assumption. It follows by (REL) that $f_{i}\left(\mathcal{D}^{N}, r\right)>0$. If $r$ is irrelevant to $j$ in $\mathcal{D}^{N}$, then $r$ remains irrelevant to $j$ in $\mathcal{D}^{\{i, j\}}$. Thus, by $(R E L), f_{j}\left(\mathcal{D}^{N}, r\right)=f_{j}\left(\mathcal{D}^{\{i, j\}}, r\right)=0$ and ( 9$)$ holds. Now suppose that $r$ is relevant to $j$ in $\mathcal{D}^{N}$. Let $M$ be the set of all agents to whom $r$ is relevant in $\mathcal{D}^{N}$. Pose $M \backslash\{i, j\}=\left\{i_{1}, i_{2}, \ldots, i_{t}\right\}$ and $M_{k}=\left\{i, j, i_{1}, i_{2}, \ldots, i_{k}\right\}$ for $1 \leq k \leq t$. Since $f_{j}\left(\mathcal{D}^{N}, r\right)=0$ for all $j \in N \backslash M$, then $(C O N)$ implies that $\left(f_{k}\left(\mathcal{D}^{N}, r\right)\right)_{k \in M}=\left(f_{k}\left(\mathcal{D}^{M}, r\right)\right)_{k \in M}$ when agents in $N \backslash M$ leave, one at a time. Note that $r$ remains relevant to both $i$ and $j$ in all sub-profiles $\mathcal{D}^{M_{k}}$ for $1 \leq k \leq t$. Therefore, by $(R E L), f_{i}\left(\mathcal{D}^{M_{k}}, r\right)>0, f_{j}\left(\mathcal{D}^{M_{k}}, r\right)>0$ and $1-f_{i}\left(\mathcal{D}^{M_{k}}, r\right)>0$. It then follows by $(C O N)$ that: 


$$
\frac{f_{j}\left(\mathcal{D}^{M}, r\right)}{f_{i}\left(\mathcal{D}^{M}, r\right)}=\frac{f_{j}\left(\mathcal{D}^{M_{k-1}}, r\right)}{f_{i}\left(\mathcal{D}^{M_{k-1}}, r\right)}=\ldots=\frac{f_{j}\left(\mathcal{D}^{\{i, j\}}, r\right)}{f_{i}\left(\mathcal{D}^{\{i, j\}}, r\right)},
$$

when agents in $M \backslash\{i, j\}$ leave, one at a time. Therefore (9) holds by recalling that $f_{j}\left(\mathcal{D}^{N}, r\right)=f_{j}\left(\mathcal{D}^{M}, r\right)$ for all $j \in M$.

It appears from Proposition 3.1 that the relative size of the cost ratio of two agents for the cost of a resource does not depend on other agents' needs when the cost ratio index under consideration is $(C O N)$.

Corollary 3.1 If two reasonable cost ratio indices $f$ and $g$ both satisfy $(C O N)$ and coincide over all profiles with two agents on the cost ratio of all resources that are not redundant, then $f$ and $g$ coincide over all profiles (with two or more agents) on the cost ratio of all resources that are not redundant.

Proof Let $f$ and $g$ be two reasonable cost ratio indices that both satisfy $(C O N)$ and coincide over all profiles with two agents on the cost ratio of all resources that are not redundant. Consider a profile $\mathcal{D}^{N}$ with two or more agents, an agent $i \in N$ and a resource $r$ in $\mathcal{D}^{N}$ that is not redundant ( $r$ is relevant to some agents in the profile).

First suppose that $r$ is relevant to $i$. Since $f$ is reasonable and satisfies $(C O N)$, then it follows from Proposition 3.1 that

$$
f_{j}\left(\mathcal{D}^{N}, r\right)=f_{i}\left(\mathcal{D}^{N}, r\right) \frac{f_{j}\left(\mathcal{D}^{\{i, j\}}, r\right)}{f_{i}\left(\mathcal{D}^{\{i, j\}}, r\right)} \text { for all } j \in N .
$$

Therefore by the budget-balance condition,

$$
\begin{aligned}
f_{i}\left(\mathcal{D}^{N}, r\right) & =\frac{1}{\sum_{j \in N} \frac{f_{j}\left(\mathcal{D}^{[i, j\}}, r\right)}{f_{i}\left(\mathcal{D}^{[i, j\}}, r\right)}} \\
& =\frac{1}{\sum_{j \in N} \frac{g_{j}\left(\mathcal{D}^{\{i, j\}}, r\right)}{g_{i}\left(\mathcal{D}^{i, j\}}, r\right)}} \\
& =g_{i}\left(\mathcal{D}^{N}, r\right) \text { since } g \text { also satisfies }(C O N)
\end{aligned}
$$

Now suppose that $r$ is irrelevant to agent $i$. By assumption, $r$ is relevant to some other agents. Then by $(R E L), f_{i}\left(\mathcal{D}^{N}, r\right)=g_{i}\left(\mathcal{D}^{N}, r\right)=0$.

Corollary 3.2 Consider a reasonable cost ratio index $f$ that satisfies $(C O N)$; a non empty set $S$ of agents; and two profiles $\mathcal{E}^{N}$ and $\mathcal{D}^{N}$ that differ only on the service constraints of agents in $S$. Then for all resources $r$ in $\mathcal{D}^{N}$ that are not redundant,

$$
\sum_{i \in S} f_{i}\left(\mathcal{D}^{N}, r\right)=\sum_{i \in S} f_{i}\left(\mathcal{E}^{N}, r\right) \Longrightarrow f_{k}\left(\mathcal{D}^{N}, r\right)=f_{k}\left(\mathcal{E}^{N}, r\right) \quad \text { for all } \quad k \in N \backslash S .
$$


Proof Let $f$ be a reasonable cost ratio that satisfies $(C O N)$. Consider two profiles $\mathcal{E}^{N}$ and $\mathcal{D}^{N}$ that differ only on the service constraints of agents in a nonempty subset $S$ of agents; that is $\mathcal{D}^{k}=\mathcal{E}^{k}$ for all $k \in N \backslash S$. Let $r$ be a resource that is not redundant in $\mathcal{D}^{N}$. Suppose that $\sum_{i \in S} f_{i}\left(\mathcal{D}^{N}, r\right)=\sum_{i \in S} f_{i}\left(\mathcal{E}^{N}, r\right)$. Given an agent $k \in N \backslash S$, there are two possible cases.

If $r$ is irrelevant to agent $k$ in $\mathcal{D}^{N}$, then $r$ is also irrelevant to $k$ in $\mathcal{E}^{N}$ and $f_{k}\left(\mathcal{D}^{N}, r\right)=f_{k}\left(\mathcal{E}^{N}, r\right)=0$ since $f$ satisfies $(R E L)$.

Now, suppose that $r$ is relevant to $k$ in $\mathcal{D}^{N}$. Then $r$ is also relevant to $k$ in $\mathcal{E}^{N}$. By $(C O N)$ and Proposition 3.1, it follows that

$$
\sum_{l \in N \backslash S} f_{l}\left(\mathcal{D}^{N}, r\right)=f_{k}\left(\mathcal{D}^{N}, r\right)\left(1+\sum_{l \in N \backslash(S \cup\{k\})} \frac{f_{l}\left(\mathcal{D}^{\{k, l\}}, r\right)}{f_{k}\left(\mathcal{D}^{\{k, l\}}, r\right)}\right)
$$

and

$$
\sum_{l \in N \backslash S} f_{l}\left(\mathcal{E}^{N}, r\right)=f_{k}\left(\mathcal{E}^{N}, r\right)\left(1+\sum_{l \in N \backslash(S \cup\{k\})} \frac{f_{l}\left(\mathcal{D}^{\{k, l\}}, r\right)}{f_{k}\left(\mathcal{D}^{\{k, l\}}, r\right)}\right)
$$

Since $\sum_{i \in S} f_{i}\left(\mathcal{D}^{N}, r\right)=\sum_{i \in S} f_{i}\left(\mathcal{E}^{N}, r\right)$, then by the budget-balance condition together with (10) and (11), $f_{k}\left(\mathcal{D}^{N}, r\right)=f_{k}\left(\mathcal{E}^{N}, r\right)$ for all $k \in N \backslash S$.

\subsection{Main result}

We now provide our main result on an alternative characterization of the counting cost ratio index $\phi$.

Theorem 3.1 A cost ratio index $f$ is reasonable and satisfies (CON), (ISI) and (IRC) if, and only if, $f$ is the counting cost ratio index; that is $f=\phi$.

The proof of Theorem 3.1 is based on four lemmas. Since it is straightforward to verify that $\phi$ is a cost ratio index that is reasonable and satisfies (CON), (ISI) and $(I R C)$, we will only prove the converse. It is worth mentioning that all the results we present from now on hold assuming that there is an infinite number of potential resources and at least three agents.

Lemma 3.1 A cost ratio index $f$ satisfies (ISI) if, and only if, for all profiles $\mathcal{D}^{N}$, for all resources $r$ in $\mathcal{D}^{N}$, for all agents $i \in N$ and for all service constraints $\mathcal{E}^{i}$ such that $\left|\overline{\mathcal{D}}^{i}\right|=\left|\overline{\mathcal{E}}^{i}\right|$ and $\left|\overline{\mathcal{D}}^{i}(r)\right|=\left|\overline{\mathcal{E}}^{i}(r)\right|, f\left(\mathcal{D}^{N}, r\right)=f\left(\left(\mathcal{E}^{i}, \mathcal{D}^{N \backslash\{i\}}\right), r\right)$.

Proof Let $f$ be a cost ratio index.

Suppose that $f$ satisfies $(I S I)$. Consider a profile $\mathcal{D}^{N}$, a resource $r$ in $\mathcal{D}^{N}$, an agent $i \in N$ and a service constraint $\mathcal{E}^{i}$ such that $\left|\overline{\mathcal{D}}^{i}\right|=\left|\overline{\mathcal{E}}^{i}\right|=q \geq 1$ and $\left|\overline{\mathcal{D}}^{i}(r)\right|=\left|\overline{\mathcal{E}}^{i}(r)\right|=p \geq 0$. Consider $q$ new resources $r_{1}, \ldots, r_{q}$ not in $\mathcal{D}^{N}$, nor in $\mathcal{E}^{i}$. 
Pose $\overline{\mathcal{D}}^{i}(r)=\left\{A_{1}, \ldots, A_{p}\right\}$ and $\overline{\mathcal{D}}^{i}=\left\{A_{1}, \ldots, A_{p}, A_{p+1}, \ldots, A_{q}\right\}$ such that $r \in A_{t}$ for $1 \leq t \leq p$ and $r \notin A_{t}$ for $p<t \leq q$. Moving from $\mathcal{D}_{0}^{i}=\mathcal{D}^{i}$ and by only adding $r_{1}$ to $A_{1}$, one obtains a new service constraint $\mathcal{D}_{1}^{i}$ such that $\overline{\mathcal{D}}_{1}^{i}=\left\{A_{1} \cup\left\{r_{1}\right\}, A_{2}, \ldots, A_{q}\right\}$. By $(I S I), f\left(\mathcal{D}^{N}, r\right)=f\left(\left(\mathcal{D}_{1}^{i}, \mathcal{D}^{N \backslash\{i\}}\right), r\right)$. Repeating this operation $q$ times while moving from $\mathcal{D}_{t}^{i}$ to $\mathcal{D}_{t+1}^{i}$ by only adding $r_{t+1}$ to $A_{t+1}$, it follows by (ISI) that $f\left(\mathcal{D}^{N}, r\right)=f\left(\left(\mathcal{D}_{q}^{i}, \mathcal{D}^{N \backslash\{i\}}\right), r\right)$. Starting from $\mathcal{D}_{q}^{i}$ and by only removing out of agent $i$ 's service constraint, one at a time, all resources in $A_{1} \cup A_{2} \cup \ldots \cup A_{q}$, it follows by (ISI) that individual shares of the cost of $r$ do not change. That is $f_{-i}\left(\left(\mathcal{D}_{q}^{i}, \mathcal{D}^{N \backslash\{i\}}\right), r\right)=f\left(\left(\mathcal{C}^{i}, \mathcal{D}^{N \backslash\{i\}}\right), r\right)$ where $\mathcal{C}^{i}$ is the service constraint such that $\overline{\mathcal{C}}^{i}=\left\{\left\{r, r_{1}\right\}, \ldots,\left\{r, r_{p}\right\},\left\{r_{p+1}\right\}, \ldots,\left\{r_{q}\right\}\right\}$. Hence $f\left(\mathcal{D}^{N}, r\right)=f\left(\left(\mathcal{C}^{i}, \mathcal{D}^{N \backslash\{i\}}\right), r\right)$. The same reasoning, when applied on $\mathcal{E}^{l}$ yields $f\left(\left(\mathcal{E}^{i}, \mathcal{D}^{N \backslash\{i\}}\right), r\right)=f\left(\left(\mathcal{C}^{i}, \mathcal{D}^{N \backslash\{i\}}\right), r\right)$. Therefore $f\left(\mathcal{D}^{N}, r\right)=f\left(\left(\mathcal{E}^{i}, \mathcal{D}^{N \backslash\{i\}}\right), r\right)$.

Conversely, suppose that for all profiles $\mathcal{D}^{N}$, for all resources $r$ in $\mathcal{D}^{N}$, for all agents $i \in N$ and for all service constraints $\mathcal{E}^{i}$ such that $\left|\overline{\mathcal{D}}^{i}\right|=\left|\overline{\mathcal{E}}^{i}\right|$ and $\left|\overline{\mathcal{D}}^{i}(r)\right|=\left|\overline{\mathcal{E}}^{i}(r)\right|, f\left(\mathcal{D}^{N}, r\right)=f\left(\left(\mathcal{E}^{i}, \mathcal{D}^{N \backslash\{i\}}\right), r\right)$. Consider two profiles $\mathcal{D}^{N}$ and $\mathcal{E}^{N}$, a resource $b$ in $\mathcal{E}^{N}$ such that for all agents $i \in N, b$ is supplementary to agent $i$ 's needs from $\mathcal{D}^{i}$ to $\mathcal{E}^{i}$ whenever $\mathcal{D}^{i} \neq \mathcal{E}^{i}$. Let $r$ be a resource in $\mathcal{D}^{N}$ such that $r \neq b$. By definition of a supplementary resource, it follows that $\left|\overline{\mathcal{D}}^{i}\right|=\left|\overline{\mathcal{E}}^{i}\right|$ and $\left|\overline{\mathcal{D}}^{i}(r)\right|=\left|\overline{\mathcal{E}}^{i}(r)\right|$ for all agents $i \in N$. Then from $\mathcal{D}^{N}$ and by only substituting, one at a time, all agents service constraints from $\mathcal{E}^{N}$ to their corresponding service constraints in $\mathcal{D}^{N}$, it follows by the assumption that individual shares of the cost of $r$ do not change. That is $f\left(\mathcal{D}^{N}, r\right)=f\left(\mathcal{E}^{N}, r\right)$.

Remark 3.1 Lemma 3.1 is a characterization of all cost ratio indices that are (ISI). The proof we present is mainly due to Hougaard and Moulin (2014, page 18); although the authors stated only an implication and used neutrality.

Lemma 3.2 If a reasonable cost ratio index $f$ satisfies (CON), (ISI) and (IRC), then for all pairs $\{i, j\}$ of agents, for all profiles $\mathcal{D}^{\{i, j\}}$, for all resources $r$ that are not redundant in $\mathcal{D}^{\{i, j\}}$ and for all finite non empty set $X$ of resources not in profile $\mathcal{D}^{\{i, j\}}$, $f\left(\mathcal{D}^{\{i, j\}}, r\right)=f\left(\left(\mathcal{D}^{i}[X], \mathcal{D}^{j}\right), r\right)$.

Proof Assume that $f$ is a reasonable cost ratio index that satisfies $(C O N),(I S I)$ and (IRC). Consider a profile $\mathcal{D}^{\{i, j\}}$, a resource $r \in \mathcal{R}\left(\mathcal{D}^{\{i, j\}}\right)$ that is not redundant in $\mathcal{D}^{\{i, j\}}$ and a finite non empty set $X=\left\{r_{1}, r_{2}, \ldots, r_{t}\right\}$ of resources not in profile $\mathcal{D}^{\{i, j\}}$, that is $X \cap \mathcal{R}\left(\mathcal{D}^{\{i, j\}}\right)=\emptyset$. By the definition of $\mathcal{D}^{i}[X], r$ is also not redundant in profile $\mathcal{E}^{\{i, j\}}=\left(\mathcal{D}^{i}[X], \mathcal{D}^{j}\right)$. If $r$ is irrelevant to agent $i$ in $\mathcal{D}^{\{i, j\}}$, then $r$ is also irrelevant to agent $i$ in $\mathcal{E}^{\{i, j\}}$. In this case, $f_{i}\left(\mathcal{D}^{\{i, j\}}, r\right)=f_{i}\left(\mathcal{E}^{\{i, j\}}, r\right)=0$ by $(R E L) ;$ and $f_{j}\left(\mathcal{D}^{\{i, j\}}, r\right)=f_{j}\left(\mathcal{E}^{\{i, j\}}, r\right)=1$ by the budget-balance condition. Hence 
$f\left(\mathcal{D}^{\{i, j\}}, r\right)=f\left(\mathcal{E}^{\{i, j\}}, r\right)$. Now assume that $r$ is relevant to agent $i$; that is $\left|\overline{\mathcal{D}}^{i}(r)\right| \geq 1$. Let $\left|\overline{\mathcal{D}}^{i}\right|=q$ and $\left|\overline{\mathcal{D}}^{i}(r)\right|=p$ for some integers $p$ and $q$ such that $q \geq p \geq 1$.

Note that for $t=1, \mathcal{D}^{i} \sim^{r}(p, q)$ and $\mathcal{D}^{i}[X] \sim^{r}(p, q)$. In this case, Lemma 3.1 implies that $f\left(\mathcal{D}^{\{i, j\}}, r\right)=f\left(\mathcal{E}^{\{i, j\}}, r\right)$. In the case $t \geq 2$, we consider a set $S=\left\{i_{1}, i_{2}, \ldots, i_{t-1}\right\}$ of $t-1$ new agents, a set $Y=\left\{r_{1}^{\prime}, r_{2}^{\prime}, \ldots, r_{(t-1) q}^{\prime}\right\}$ of $(t-1) \times q$ new resources and pose for all $s \in\{1,2, \ldots, t-1\}$,

$$
\mathcal{D}^{i_{s}}=\left\{\left\{r, r_{(s-1) q+1}^{\prime}\right\},\left\{r, r_{(s-1) q+2}^{\prime}\right\}, \ldots,\left\{r, r_{(s-1) q+p}^{\prime}\right\},\left\{r_{(s-1) q+p+1}^{\prime}\right\}, \ldots,\left\{r_{s q}^{\prime}\right\}\right\}
$$

Each service constraint $\mathcal{D}^{i_{s}}$ satisfies $\mathcal{D}^{i_{s}} \sim^{r}(p, q)$. Moreover, the needs of $\mathcal{D}^{\left\{i, i_{1}, i_{2}, \ldots, i_{t-1}\right\}}$ of agents in $S^{\prime}=\left\{i, i_{1}, i_{2}, \ldots, i_{t-1}\right\}$ are mergeable and have the same types. Thus the concatenation $\mathcal{C}=\mathcal{D}^{\left[\left\{i, i_{1}, i_{2}, \ldots, i_{t-1}\right\}\right]}$ of the needs of agents in $S^{\prime}$ is regular. It follows that

$$
\begin{aligned}
\frac{f_{j}\left(\mathcal{D}^{\{i, j\}}, r\right)}{f_{i}\left(\mathcal{D}^{\{i, j\}}, r\right)}= & \frac{f_{j}\left(\left(\mathcal{D}^{\{i, j\}}, \mathcal{D}^{i_{1}}, \mathcal{D}^{i_{2}}, \ldots, \mathcal{D}^{i_{t-1}}\right), r\right)}{f_{i}\left(\left(\mathcal{D}^{\{i, j\}},, \mathcal{D}^{i_{1}}, \mathcal{D}^{i_{2}}, \ldots, \mathcal{D}^{i_{t-1}}\right), r\right)} \quad \text { by Proposition } 3.1 \\
& =\frac{f_{j}\left(\left(\mathcal{C}, \mathcal{D}^{j}, \mathcal{C}, \mathcal{C}, \ldots, \mathcal{C}\right), r\right)}{f_{i}\left(\left(\mathcal{C}, \mathcal{D}^{j}, \mathcal{C}, \mathcal{C}, \ldots, \mathcal{C}\right), r\right)} \quad \text { by }(I R C) \text { since } \mathcal{C} \text { is a regular concatenation } \\
& =\frac{f_{j}\left(\left(\mathcal{C}, \mathcal{D}^{j}\right), r\right)}{f_{i}\left(\left(\mathcal{C}, \mathcal{D}^{j}\right), r\right)} \quad \text { by Proposition } 3.1 \\
& =\frac{f_{j}\left(\left(\mathcal{D}^{i}[X], \mathcal{D}^{j}\right), r\right)}{f_{i}\left(\left(\mathcal{D}^{i}[X], \mathcal{D}^{j}\right), r\right)}
\end{aligned}
$$

by Lemma 3.1 since $\mathcal{D}^{i}[X] \sim^{r}(t p, t q)$ and $\mathcal{C} \sim^{r}(t p, t q)$

By the budget-balance condition applied to $f\left(\mathcal{D}^{\{i, j\}}, r\right)$ and $f\left(\left(\mathcal{D}^{i}[X], \mathcal{D}^{j}\right), r\right)$, we get $f\left(\mathcal{D}^{\{i, j\}}, r\right)=f\left(\left(\mathcal{D}^{i}[X], \mathcal{D}^{j}\right), r\right)$.

Lemma 3.3 Iff is a reasonable cost ratio index that satisfies (CON), (ISI) and (IRC), then $f\left(\mathcal{D}^{\{i, j\}}, r\right)=\phi\left(\mathcal{D}^{\{i, j\}}, r\right)$ for all pairs $\{i, j\}$ of agents, for all profiles $\mathcal{D}^{\{i, j\}}$ and for all resources $r$ such that $\left|\overline{\mathcal{D}}^{i}\right|=\left|\overline{\mathcal{D}}^{j}\right| \geq 1$ and $\left|\overline{\mathcal{D}}^{i}(r)\right|=1$.

Proof Assume that $f$ is a reasonable cost ratio index that meets $(C O N),(I S I)$ and $(I R C)$. Consider a profile $\mathcal{D}^{\{i, j\}}$ and a resource $r$ such that $\left|\overline{\mathcal{D}}^{i}\right|=\left|\overline{\mathcal{D}}^{j}\right|=q,\left|\overline{\mathcal{D}}^{i}(r)\right|=1$ and $\left|\overline{\mathcal{D}}^{j}(r)\right|=p \geq 0$. If $r$ is irrelevant to agent $j$, then $f\left(\mathcal{D}^{\{i, j\}}, r\right)=\phi\left(\mathcal{D}^{\{i, j\}}, r\right)$ by (REL) and the budget-balance condition. Now assume that $r$ is relevant to agent $j$; that is $p \geq 1$. To show that $f\left(\mathcal{D}^{\{i, j\}}, r\right)=\phi\left(\mathcal{D}^{\{i, j\}}, r\right)$, we proceed by induction on $\left|\overline{\mathcal{D}}^{j}(r)\right|=p \in\{1,2, \ldots, q\}$. 
First suppose that $p=1$. Then by Lemma 3.1, $f\left(\mathcal{D}^{\{i, j\}}, r\right)=f\left(\mathcal{E}^{\{i, j\}}, r\right)$ where $\mathcal{E}^{i}=\mathcal{E}^{j}=\mathcal{D}^{i}$. Since $f$ is reasonable, then $f_{i}\left(\mathcal{E}^{\{i, j\}}, r\right)=f_{j}\left(\mathcal{E}^{\{i, j\}}, r\right)$ by (IDE). Thus $f\left(\mathcal{E}^{\{i, j\}}, r\right)=\left(\frac{1}{2}, \frac{1}{2}\right)=\phi\left(\mathcal{E}^{\{i, j\}}, r\right)$. Hence $f\left(\mathcal{D}^{\{i, j\}}, r\right)=\phi\left(\mathcal{D}^{\{i, j\}}, r\right)=\left(\frac{1}{2}, \frac{1}{2}\right)$.

Now consider $p \in\{2, \ldots, q\}$ and suppose that $f\left(\mathcal{D}^{\{i, j\}}, r\right)=\phi\left(\mathcal{D}^{\{i, j\}}, r\right)$ whenever $\left|\mathcal{D}^{j}(r)\right|<p$. Suppose that $\left|\mathcal{D}^{j}(r)\right|=p$. There are two possible cases depending on whether $p$ is odd or even. In both cases, $p=2 p^{\prime}+\varepsilon$ where $\varepsilon=0$ or $\varepsilon=1$. Consider a third agent $k$, and three service constraints $\mathcal{E}^{k}, \mathcal{F}^{j}$ and $\mathcal{F}^{k}$ such that $\overline{\mathcal{D}}^{j} \cap \overline{\mathcal{E}}^{k}=\emptyset$, $\left|\overline{\mathcal{E}}^{k}\right|=q, \quad\left|\overline{\mathcal{E}}^{k}(r)\right|=\varepsilon, \quad$ while $\quad \overline{\mathcal{F}}^{j} \cap \overline{\mathcal{F}}^{k}=\emptyset, \quad\left|\overline{\mathcal{F}}^{i}\right|=\left|\overline{\mathcal{F}}^{k}\right|=q \quad$ and $\left|\overline{\mathcal{F}}^{j}(r)\right|=\left|\overline{\mathcal{F}}^{k}(r)\right|=p^{\prime}+\varepsilon$. Furthermore, let $X=\left\{r_{1}, r_{2}\right\}$ be a set of two new identical resources. Pose $\mathcal{T}^{j}=\mathcal{F}^{j}[X]$ and $\mathcal{T}^{k}=\mathcal{F}^{k}[X]$. By definition, $\mathcal{T}^{j} \sim^{r}\left(2 p^{\prime}+2 \varepsilon, 2 q\right)$ and $\mathcal{T}^{k} \sim^{r}\left(2 p^{\prime}+2 \varepsilon, 2 q\right)$. It follows that

$$
\begin{aligned}
\frac{f_{i}\left(\mathcal{D}^{\{i, j\}}, r\right)}{f_{j}\left(\mathcal{D}^{\{i, j\}}, r\right)}= & \frac{f_{i}\left(\left(\mathcal{D}^{\{i, j\}}, \mathcal{E}^{k}\right), r\right)}{f_{j}\left(\left(\mathcal{D}^{\{i, j\}}, \mathcal{E}^{k}\right), r\right)} \text { by Proposition } 3.1 \\
= & \frac{f_{i}\left(\left(\mathcal{D}^{i},\left[\mathcal{D}^{j} \mathcal{E}^{k}\right],\left[\mathcal{D}^{j} \mathcal{E}^{k}\right]\right), r\right)}{f_{j}\left(\left(\mathcal{D}^{i},\left[\mathcal{D}^{j} \mathcal{E}^{k}\right],\left[\mathcal{D}^{j} \mathcal{E}^{k}\right]\right), r\right)} \\
& \text { by }(I R C) \text { via a concatenation of } \mathcal{D}^{j} \text { and } \mathcal{E}^{k} \\
= & \frac{f_{i}\left(\left(\mathcal{D}^{i}, \mathcal{T}^{j}, \mathcal{T}^{k}\right), r\right)}{f_{j}\left(\left(\mathcal{D}^{i}, \mathcal{T}^{j}, \mathcal{T}^{k}\right), r\right)} \\
& \text { by Lemma } 3.1 \text { since }\left[\mathcal{D}^{j} \mathcal{E}^{k}\right] \sim^{r}\left(2\left(p^{\prime}+\varepsilon\right), 2 q\right) \\
= & \frac{f_{i}\left(\left(\mathcal{D}^{i}, \mathcal{F}^{j}, \mathcal{F}^{k}\right), r\right)}{f_{j}\left(\left(\mathcal{D}^{i}, \mathcal{F}^{j}, \mathcal{F}^{k}\right), r\right)}
\end{aligned}
$$

by Lemma 3.1 since $\mathcal{T}^{j}=\mathcal{F}^{j}[X]$ and $\mathcal{T}^{k}=\mathcal{F}^{k}[X]$

$$
\begin{aligned}
& =\frac{f_{i}\left(\left(\mathcal{D}^{i}, \mathcal{F}^{j}\right), r\right)}{f_{j}\left(\left(\mathcal{D}^{i}, \mathcal{F}^{j}\right), r\right)} \text { by Proposition } 3.1 \\
& =\frac{\phi_{i}\left(\left(\mathcal{D}^{i}, \mathcal{F}^{j}\right), r\right)}{\phi_{j}\left(\left(\mathcal{D}^{i}, \mathcal{F}^{j}\right), r\right)} \text { by induction assumption }
\end{aligned}
$$

Noting that $\frac{\phi_{i}\left(\left(\mathcal{D}^{i}, \mathcal{F}^{j}\right), r\right)}{\phi_{j}\left(\left(\mathcal{D}^{i}, \mathcal{F}^{j}\right), r\right)}=\frac{\phi_{i}\left(\mathcal{D}^{\{i, j\}}, r\right)}{\phi_{j}\left(\mathcal{D}^{\{i, j\}}, r\right)}=\frac{1}{p^{\prime}+\varepsilon}$, we deduce by the budget-balance condition that $f\left(\mathcal{D}^{\{i, j\}}, r\right)=\phi\left(\mathcal{D}^{\{i, j\}}, r\right)$. This completes the proof.

Lemma 3.4 If $f$ is a reasonable cost ratio index that meets (CON), (ISI) and (IRC), then $f\left(\mathcal{D}^{\{i, j\}}, r\right)=\phi\left(\mathcal{D}^{\{i, j\}}, r\right)$ for all pairs $\{i, j\}$ of agents, for all profiles $\mathcal{D}^{\{i, j\}}$ and for all resource $r$ in $\mathcal{D}^{\{i, j\}}$ that are not redundant. 
Proof Assume that $f$ is a reasonable cost ratio index that satisfies $(C O N),(I S I)$ and $(I R C)$. Consider a profile $\mathcal{D}^{\{i, j\}}$ and a resource $r$ in $\mathcal{D}^{\{i, j\}}$. Let $\left|\overline{\mathcal{D}}^{i}\right|=q_{i},\left|\overline{\mathcal{D}}^{j}\right|=q_{j}$, $\left|\overline{\mathcal{D}}^{i}(r)\right|=p_{i} \geq 0 \quad$ and $\quad\left|\overline{\mathcal{D}}^{j}(r)\right|=p_{j} \geq 0 . \quad$ Let $\quad X=\left\{x_{1}, x_{2}, \ldots, x_{q_{j}}\right\} \quad$ and $Y=\left\{y_{1}, y_{2}, \ldots, y_{q_{i}}\right\}$ be two disjoint sets of identical resources.

Since $r$ is not redundant, $p_{i} \geq 1$ or $p_{j} \geq 1$. Without lost of generality, assume that $p_{i} \geq 1$. Pose $\mathcal{E}^{i}=\mathcal{D}^{i}[X]$ and $\mathcal{E}^{j}=\mathcal{D}^{j}[Y]$. By the definition of $\mathcal{D}^{i}[X]$ and $\mathcal{D}^{j}[Y]$, $\left|\overline{\mathcal{E}}^{i}\right|=\left|\overline{\mathcal{E}}^{j}\right|=q_{i} q_{j},\left|\overline{\mathcal{D}}^{i}(r)\right|=q_{j} p_{i}$ and $\left|\overline{\mathcal{D}}^{j}(r)\right|=q_{i} p_{j}$. Consider an agent $k \notin\{i, j\}$ and a service constraint $\mathcal{E}^{k}$ such that $\left|\overline{\mathcal{E}}^{k}\right|=q_{i} q_{j}$ and $\left|\overline{\mathcal{E}}^{k}(r)\right|=1$. By Proposition 3.1, $\frac{f_{l}\left(\mathcal{E}^{\{i, j, k\}}, r\right)}{f_{k}\left(\mathcal{E}^{[i, j, k\}}, r\right)}=\frac{f_{l}\left(\mathcal{E}^{[l, k\}}, r\right)}{f_{k}\left(\mathcal{E}^{[l, k\}}, r\right)}$ for all $l \in\{i, j\}$. Recalling that $\mathcal{E}^{i}=\mathcal{D}^{i}[X]$ and $\mathcal{E}^{j}=\mathcal{D}^{j}[Y]$, Lemma 3.2 implies that $f\left(\mathcal{E}^{\{i, j\}}, r\right)=f\left(\left(\mathcal{E}^{i}, \mathcal{D}^{j}\right), r\right)=f\left(\mathcal{D}^{\{i, j\}}, r\right)$. By Lemma 3.3, $\frac{f_{i}\left(\mathcal{E}^{\{i, k\}}, r\right)}{f_{k}\left(\mathcal{E}^{[i, k\}}, r\right)}=\frac{\phi_{i}\left(\mathcal{E}^{\{i, k\}}, r\right)}{\phi_{k}\left(\mathcal{E}^{[i, k\}}, r\right)}=q_{j} p_{i}$ and $\frac{f_{j}\left(\mathcal{E}^{[j, k\}}, r\right)}{f_{k}\left(\mathcal{E}^{[j, k\}}, r\right)}=\frac{\phi_{j}\left(\mathcal{E}^{[j, k\}}, r\right)}{\phi_{k}\left(\mathcal{E}^{[j, k\}}, r\right)}=q_{i} p_{j}$. Summing individual shares of the cost of resource $r$ in profile $\mathcal{E}^{\{i, j, k\}}$ leads by the budget-balance condition to $f_{k}\left(\mathcal{E}^{\{i, j, k\}}, r\right)=\frac{1}{q_{j} p_{i}+q_{i} p_{j}+1}, \quad f_{i}\left(\mathcal{E}^{\{i, j, k\}}, r\right)=\frac{q_{j} p_{i}}{q_{j} p_{i}+q_{i} p_{j}+1} \quad$ and

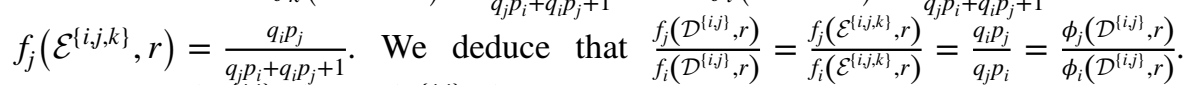
Therefore, $f\left(\mathcal{D}^{\{i, j\}}, r\right)=\phi\left(\mathcal{E}^{\{i, j\}}, r\right)$ by the budget-balance condition.

Taking advantage of the previous lemmas, a proof of Theorem 3.1 is the following:

Proof (Proof of Theorem 3.1) Assume that $f$ is reasonable, $(C O N),(I S I)$ and (IRC). Consider a profile $\mathcal{D}^{N}$ with two or more agents, an agent $i \in N$ and a resource $r$ in $\mathcal{D}^{N}$.

First suppose that $r$ is not redundant in $\mathcal{D}^{N}$. Then by Lemma 3.4, $f$ coincides with $\phi$ over all profiles with two agents on the cost ratio of all resources that are not redundant. Since $f$ and $\phi$ both satisfy $(C O N)$, then $f\left(\mathcal{D}^{N}, r\right)=\phi\left(\mathcal{D}^{N}, r\right)$ by Corollary 3.1.

Now, suppose that $r$ is redundant in $\mathcal{D}^{N}$. Pose $\left|\overline{\mathcal{D}}^{i}\right|=q_{i}$, for $i \in N$. Consider a new resource $r^{\prime}$ and for all $i \in N$, let $X^{i}=\left\{x_{1}, x_{2}, \ldots, x_{q_{i}}\right\}$ be a set of $q_{i}$ new identical resources other than $r^{\prime}$. Let $\mathcal{E}^{N}, \mathcal{F}^{N}$ and $\mathcal{T}^{N}$ be three profiles such that for all $i \in N$, $\overline{\mathcal{E}}^{i}=\left\{\left\{r^{\prime}\right\}\right\}, \overline{\mathcal{F}}=\left\{\left\{x_{t}\right\}: 1 \leq t \leq q_{i}\right\}$ and $\overline{\mathcal{T}}^{i}=\left\{\left\{r^{\prime}, x_{t}\right\}: 1 \leq t \leq q_{i}\right\}$. Note that for $Y=\left\{r^{\prime}\right\}, \mathcal{T}^{\dot{i}}=\mathcal{E}^{i}\left[X^{i}\right]$ and $\mathcal{T}^{\dot{i}}=\mathcal{F}^{i}[Y]$. Since $f$ is reasonable, $f\left(\mathcal{T}^{i, j\}}, r\right)=\left(\frac{1}{2}, \frac{1}{2}\right)$. It follows that

$$
\begin{aligned}
f\left(\mathcal{D}^{N}, r\right) & =f\left(\left(\mathcal{F}^{i}, \mathcal{D}^{N \backslash\{i\}}\right), r\right) \text { by Lemma } 3.1 \text { since } \mathcal{D}^{i} \sim^{r}\left(0, q_{i}\right) \text { and } \mathcal{F}^{i} \sim^{r}\left(0, q_{i}\right) \\
& =f\left(\left(\mathcal{T}^{i}, \mathcal{D}^{N \backslash\{i\}}\right), r\right) \text { by Lemma } 3.2 \text { since } \mathcal{T}^{i}=\mathcal{F}^{i}[Y] \\
& =f\left(\left(\mathcal{E}^{i}, \mathcal{D}^{N \backslash\{i\}}\right), r\right) \text { by Lemma } 3.2 \text { since } \mathcal{T}^{\dot{i}}=\mathcal{E}^{i}\left[X^{i}\right]
\end{aligned}
$$


This proves that replacing the service constraints $\mathcal{D}^{i}$ of agent $i$ by $\mathcal{E}^{i}$ does not affect the cost ratio for resource $r$ for any agent. Therefore, by performing $n$ times this operation, we obtain $f\left(\mathcal{D}^{N}, r\right)=f\left(\mathcal{E}^{N}, r\right)$. Moreover, all agents in $\mathcal{E}^{N}$ have the same needs. $\quad$ Therefore $f\left(\mathcal{E}^{N}, r\right)=\left(\frac{1}{n}, \frac{1}{n}, \ldots, \frac{1}{n}\right)$ Hence $f\left(\mathcal{D}^{N}, r\right)=\left(\frac{1}{n}, \frac{1}{n}, \ldots, \frac{1}{n}\right)=\phi\left(\mathcal{D}^{N}, r\right)$.

In both cases, $f\left(\mathcal{D}^{N}, r\right)=\phi\left(\mathcal{D}^{N}, r\right)$.

Independence among axioms: We prove that one cannot drop any of the five axioms in Theorem 3.1 without altering its validity.

1. Consider the cost ratio index $\lambda^{*}$ defined by

$$
\lambda_{i}^{*}\left(\mathcal{D}^{N}, r\right)=\frac{\lambda_{i}\left(\mathcal{D}^{N}, r\right)}{\sum_{j \in N} \lambda_{j}\left(\mathcal{D}^{N}, r\right)}
$$

where

$$
\lambda_{i}\left(\mathcal{D}^{N}, r\right)=\frac{\sum_{A \in \overline{\mathcal{D}}^{i}(r)}|A|}{\left|\overline{\mathcal{D}}^{i}\right|}
$$

if $r$ is relevant to agent $i ; \lambda_{i}\left(\mathcal{D}^{N}, r\right)>0$ if $r$ is redundant; and $\lambda_{i}\left(\mathcal{D}^{N}, r\right)=0$ otherwise. Then $\lambda^{*}$ is a reasonable cost ratio index that satisfies $(C O N)$ and (IRC); but not (ISI). One cannot drop (ISI).

2. Define the cost ratio index $g$ by $g_{i}\left(\mathcal{D}^{N}, r\right)=\frac{1}{|N(r)|}$ if $r$ is relevant to agent $i$; $g_{i}\left(\mathcal{D}^{N}, r\right)=\frac{1}{|N|}$ if $r$ is redundant; and $g_{i}\left(\mathcal{D}^{N}, r\right)=0$ otherwise. Then $g$ is a reasonable cost ratio index that satisfies (ISI) and $(C O N)$; but not $(I R C)$. One can not drop (IRC).

3. Let $h$ be the cost ratio index defined by $h\left(\mathcal{D}^{N}, r\right)=\frac{1}{|N|}$ if $|N| \leq 2$; and $h\left(\mathcal{D}^{N}, r\right)=\phi\left(\mathcal{D}^{N}, r\right)$ otherwise. Then $h$ is a reasonable cost ratio index that satisfies (ISI) and (IRC); but not (CON). One can not drop (CON).

4. Consider a collection $\left(w_{i}\right)_{i \in \mathcal{N}}$ of positive weights such that $w_{k} \neq w_{l}$ for some pairs $\{k, l\}$ of agents. Define the cost ratio index $w^{*}$ by

$$
w_{i}^{*}\left(\mathcal{D}^{N}, r\right)=\frac{\mathcal{W}_{i}\left(\mathcal{D}^{N}, r\right)}{\sum_{j \in N} \mathcal{W}_{j}\left(\mathcal{D}^{N}, r\right)}
$$

where $\mathcal{W}_{i}\left(\mathcal{D}^{N}, r\right)=w_{i} \theta_{j}^{1}\left(\mathcal{D}^{N}, r\right)$ if $r$ is relevant to agent $i ; \mathcal{W}_{i}\left(\mathcal{D}^{N}, r\right)=1$ if $r$ is redundant; and $\mathcal{W}_{i}\left(\mathcal{D}^{N}, r\right)=0$ otherwise. Then $w^{*}$ is $(R E L),(I S I),(C O N)$ and $(I R C)$; but not (IDE). One can not drop (IDE).

5. The cost ratio index $E$ defined by $E\left(\mathcal{D}^{N}, r\right)=\frac{1}{|N|}$ is $(I D E),(I S I),(C O N)$ and $(I R C)$; but not $(R E L)$. One can not drop (REL). 
Noting that $\phi$ is the cost ratio index associated with the counting rule, the following holds:

Corollary 3.3 Among cost allocation rules that are additive in cost, the counting rule $\Phi$ is the unique rule associated to a reasonable cost ratio index that satisfies (CON), (ISI) and (IRC).

It is worth noticing that, for cost allocation rules that are additive in cost, axioms in Corollary 3.3 are independent. Furthermore, all the five other axioms are stated under additivity. Thus, additivity itself can not be dropped in Corollary 3.3.

Concatenating or splitting some service constraints by agents may be seen as a manipulation attempt. In this case, it is desirable for a cost ratio index not to allow such attempts to be profitable. We assume that only regular concatenations are feasible. This may be dictated by some compatibility requirements in the network. For example, it may happen that only agents using similar technologies, that correspond to service constraints of the same type, can exchange.

No Advantageous Regular Concatenation (NARC): For all profiles $\mathcal{D}^{N}$, for all non empty subsets $S$ of agents having mergeable service constraints of the same type,

$$
\sum_{i \in S} f_{i}\left(\mathcal{D}^{N[S]}, r\right)=\sum_{i \in S} f_{i}\left(\mathcal{D}^{N}, r\right)
$$

for all resources $r \in \mathcal{R}\left(\mathcal{D}^{N}\right)$.

Any regular concatenation of the service constraints of some agents leaves their total shares unchanged over each possible resource.

Proposition 3.2 Let f be a reasonable cost ratio index.

1. Iff satisfies (IRC), then $f$ satisfies (NARC).

2. Iff satisfies (CON) and (NARC), then $f$ satisfies (IRC).

Proof Let $f$ be a reasonable cost ratio index.

Suppose that $f$ satisfies $(I R C)$. Consider a profile $\mathcal{D}^{N}$, a resource $r$ in $\mathcal{D}^{N}$ and a non empty set $S$ of agents having mergeable service constraints of the same type. By (IRC), $f_{k}\left(\mathcal{D}^{N[S]}, r\right)=f_{k}\left(\mathcal{D}^{N}, r\right)$ for all $k \in N \backslash S$. Recalling that individual cost ratios for $r$ sum to 1, (12) holds. Thus $f$ satisfies (NARC).

Conversely, suppose that $f$ meets $(C O N)$ and $(N A R C)$. Consider a profile $\mathcal{D}^{N}$, a resource $r$ in $\mathcal{D}^{N}$ and a non empty set $S$ of agents having mergeable service constraints of the same type. Since (12) holds by (NARC), then by Corollary 3.2, it follows that $f_{k}\left(\mathcal{D}^{N}, r\right)=f_{k}\left(\mathcal{D}^{N[S]}, r\right)$. Therefore, $f$ satisfies $(I R C)$.

By combining Theorem 3.1 and Proposition 3.2, 
Proposition 3.3 A reasonable cost ratio index f satisfies (CON), (ISI) and (NARC) if, and only if, $f=\phi$.

Proof If $f$ is a reasonable cost ratio index that satisfies $(C O N),(I S I)$ and (NARC), then by item (2.) in Proposition 3.2, $f$ also satisfies (IRC). Hence by Theorem 3.1, $f=\phi$.

Conversely, if $f=\phi$, then by Theorem $3.1, f$ is a reasonable cost ratio index that satisfies $(C O N),(I S I)$ and (IRC). By Proposition 3.2, $f$ also satisfies (NARC).

Corollary 3.4 A cost allocation rule $F$ is additive and is associated to a reasonable cost ratio index that satisfies (CON), (ISI) and (NARC) if, and only if, $F=\Phi$.

\section{Conclusion}

The counting rule and its cost ratio index for cost allocation problems with possibly redundant resources have been characterized in this paper within the class of cost allocation rules that are additive in cost. This has been achieved by combining some normative conditions such as consistency and independence properties. The intuition of independence axioms in this paper is based on the idea that, if some changes occur in the service constraints of two or more agents in such a way that the overall service demand on a resource is preserved, then other agents should not be impacted. The counting rule is not manipulable when only regular concatenations are feasible; but is manipulable in general by merging or splitting agents needs. Designing reasonable and non manipulable allocation rules within the current framework deserves further investigations.

Acknowledgements The authors gratefully acknowledge very useful comments and suggestions from Jens Leth Hougaard on an earlier version of this paper. We are also deeply grateful to the Associate Editor and reviewers for their helpful and detailed comments, to Boniface Mbih and the members of the ERMASS monthly seminar at The University of Yaounde I (Cameroon) for their encouragements.

\section{References}

Béal S, Ferrières S, Rémila E, Solal P (2018) The proportional Shapley value and applications. Games Econ Behav 108:93-112

Bergantiños G, Gómez-Rúa M, Llorca N, Pulido M, Sánchez-Soriano J (2014) A new rule for source connection problems. Eur J Oper Res 234(3):780-788

Bergantiños G, Martínez R (2014) Cost allocation in asymmetric trees. Eur J Oper Res 237(3):975-987

de Frutos MA (1999) Coalitional manipulations in a bankruptcy problem. Rev Econ Des 4(3):255-272

Hougaard JL (2009) An introduction to allocation rules. Springer, New York

Hougaard JL (2018) Sharing the costs of access to a set of public goods. In: Game theory in management accounting, pp 287-300, Springer

Hougaard JL, Moulin H (2014) Sharing the cost of redundant items. Games Econ Behav 87:339-352 
Moulin H (2000) Priority rules and other asymmetric rationing methods. Econometrica 68(3):643-684

Moulin H (2002) Axiomatic cost and surplus sharing. Handb Soc Choice Welf 1:289-357

Moulin H (2013) Cost sharing in networks: some open questions. Int Game Theory Rev 15(02):1340001

Moulin H, Laigret F (2011) Equal-need sharing of a network under connectivity constraints. Games Econ Behav 72(1):314-320

Shapley LS (1953) A value for n-person games. Contrib Theory Games 2(28):307-317

Sharkey WW (1995) Network models in economics. Handb Oper Res Manag Sci 8:713-765

Thomson W (2001) On the axiomatic method and its recent applications to game theory and resource allocation. Soc Choice Welf 18(2):327-386

Thomson W et al (2007) Cost allocation and airport problems. Rochester Center for Economic Research, Working Paper, (538)

Young HP (1988) Distributive justice in taxation. J Econ Theory 44(2):321-335

Publisher's Note Springer Nature remains neutral with regard to jurisdictional claims in published maps and institutional affiliations. 